\title{
411 INNATE: IMMUNOTHERAPY DURING NEOADJUVANT THERAPY FOR RECTAL CANCER TO ELUCIDATE LOCAL AND SYSTEMIC THERAPEUTIC RESPONSES
}

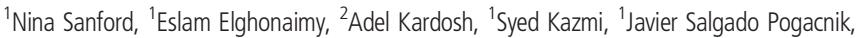
${ }^{3}$ Xiaodong Yang, 'Robert Timmerman, 'Todd Aguilera*. 'UT Southwestern, Dallas, TX, USA; ${ }^{2} \mathrm{OHSU}$, Portland, OR, USA; ${ }^{3}$ Apexigen Inc, Redwood Shores, CA, USA

Background The relative risk of developing rectal cancer has quadrupled in young adults over the last 40 years and approximately $50 \%$ of patients develop recurrence within 3 years. Thus, there is a critical need for new approaches to improve survival but cancer Immunotherapy has had little impact on colorectal cancer. The anti-CD40 agonist immunotherapy is emerging and $\mathrm{APX} 005 \mathrm{M}$ has shown promise in phase I and ongoing phase II trials. Anti-CD40 can stimulate both innate and adaptive immune responses and a greater response can be achieved combining anti-CD40 with radiation therapy (RT) in animal models. We developed the INNATE trial, a phase II randomized trial of neoadjuvant short course RT followed by chemotherapy with or without the addition of APX005M for rectal cancer (NCT04130854).

Methods The INNATE trial is a multi-center, 58 patient, 3:2 randomization clinical trial, that adds APX005M to short course RT (SCRT) and subsequent FOLFOX chemotherapy prior to definitive radiation. Eligibility includes stage III and high risk stage II rectal cancer and candidates for standard neoadjuvant therapy and no contraindications for immunotherapy or radiation. Patients receive $5 \mathrm{~Gy}$ x 5 fractions over five days and if randomized to experimental arm will receive APX005M on day 3 of radiation. After a two week break patients receive an optional endoscopy and biopsy followed by standard FOLFOX chemotherapy with APX005M infusion after disconnection of 5-FU chemotherapy pump. Study arm receives APX005M with 5 of 6 cycles of FOLFOX. After treatment patients undergo restating, endoscopy, and trans abdominal resection. The primary endpoint is pathologic complete response with the null estimated to be $20 \%$ and alternative $50 \%$ for a power of 0.8 and type 1 error of 0.1 . Secondary endpoints include overall survival, toxicity analysis, and disease free survival. For correlative analysis, tissue is collected pre-treatment, two weeks after RT, and at surgery, then blood collected during treatment and at follow up.

Results To date 16 patients have been randomized and initiated treatment. The treatment is well tolerated. Fiveteen patients received pre- and post-SCRT biopsies. The study team plans correlative analysis including single cell RNA sequencing, multiplex imunohistochemistry, and bulk sequencing. Preliminary data shows changes in the immune tumor microenvironment from patients treated as their own control and between SCRT versus SCRT + APX00M.

Conclusions The INNATE trial shows feasibility of incorporating novel immunotherapies with an emerging standard of care incorporating short course RT. It serves as an important platform for scientific and clinical investigation.

Trial Registration ClinicalTrials.gov: NCT04130854

Ethics Approval The clinical trial has institutional review board approval at the University of Texas Southwestern, Oregon Health and Sciences University, Wake Forest, and Moffitt. All patients have provided informed consent.

http://dx.doi.org/10.1136/jitc-2021-SITC2021.411 